INFORMES

\title{
Comentario sobre la sentencia del Tribunal Arbitral en el caso Eli Lilly y Compañía con el Gobierno de Canadá
}

\author{
Commentary regarding the final award of the Arbitration Tribunal \\ in the case of Eli Lilly and Company with the Government of Canada
}

\author{
Cristián BARros MiCHell \\ Abogado, Chile
}

RESUMEN Este ensayo comenta y analiza la sentencia del Tribunal Arbitral en el caso de Eli Lilly y Compañía con el Gobierno de Canadá. El trabajo se centra en el debate que existió respecto de la nulidad de dos patentes de invención de Eli Lilly en Canadá y si es que la aplicación de la denominada doctrina de la promesa de la utilidad fue un cambio drástico en la ley de Canadá, al mismo tiempo que analiza el tema de las expectativas legítimas de un titular de patentes, de la armonización del derecho de patentes en el derecho internacional y si es que Canadá incurrió en actos arbitrarios y discriminatorios al anular dichas patentes farmacéuticas del demandante.

PALABRAS CLAVE Nulidad de patentes, utilidad, doctrina de la promesa de la utilidad, armonización del derecho de patentes en el derecho internacional, cambio drástico de la ley, derecho de patentes.

ABSTRACT This essay comments and analyzes the decision rendered by the Arbitral Tribunal in the case of Eli Lilly and Company with the Government of Canada. The work centers on the debate that existed regarding the cancelation of two of Eli Lilly's patents in Canada and whether the application of the doctrine of the promise of utility was a drastic change in the law in Canada, as well as analyzing the issues of legitimate expectations of a patent holder, harmonization of international patent law and whether Canada incurred in arbitrary and discriminatory actions by canceling the two patents.

KEYWORDS Patent cancelation action, utility, doctrine of the promise of utility, harmonization of international patent law, drastic change in the law, patents law. 


\section{Introducción}

Eli Lilly and Company y su subsidiaria Eli Lilly Canadá Inc. presentaron el 12 de septiembre de 2013 una notificación de arbitraje ante un Tribunal Arbitral, fundada en el capítulo 11 sobre inversiones del Tratado de Libre Comercio de América del Norte (TLCAN).

El fundamento de Eli Lilly fue que Canadá anuló sus patentes que protegían los medicamentos Strattera (en 2010) y Zyprexa (en 2011) empleando una doctrina denominada "promesa de la utilidad», la cual sería un cambio drástico a la ley, además de arbitraria y discriminatoria en contra de las empresas farmacéuticas.

Asimismo, sostuvo que, al aplicar dicha doctrina, Canadá infringía sus obligaciones internacionales respecto de las patentes establecidas en el capítulo 17 del TLCAN y que el aplicarla en forma retroactiva constituye una expropiación ilegal de sus inversiones de acuerdo al artículo 1.110 del TLCAN, junto con una violación a su obligación de proveer un trato de acuerdo a los estándares mínimos (trato justo y equitativo) del artículo 1.105 del TLCAN (el estándar de un «trato justo y equitativo» incluye la ausencia de arbitrariedad y de no discriminación y las expectativas legítimas de las partes) (Billingsley, 2015: 32).

Canadá argumentó que el Tribunal debía rechazar la demanda por las siguientes razones: i) el único fundamento por el cual se puede argumentar que una decisión judicial de un país produce una infracción al capítulo 11 del TLCAN es la «negación de justicia», causal que no fue invocada por Eli Lilly; ii) el Tribunal no tiene competencia por razones de ratione temporis; iii) las cortes canadienses no han modificado en forma dramática su interpretación y aplicación del requisito de utilidad, y iv) la nulidad de una patente de invención no constituye una infracción al capítulo 17 del TLCAN.

$\mathrm{Si}$ bien hay distintos temas interesantes a ser analizados en esta sentencia, ${ }^{1}$ centraremos los comentarios en al ámbito de la propiedad industrial, específicamente de las patentes de invención y el requisito de utilidad.

\section{Las patentes de Zyprexa y de Strattera}

La primera patente de Eli Lilly en ser anulada fue la de Zyprexa (el principio activo es olanzapina). En 1975, Eli Lilly solicitó una patente en Canadá que protegía a millones de compuestos químicos, incluyendo a olanzapina, la cual fue concedida en 1980 (patente 1.075.687).

Posteriormente, el 24 de abril de 1991, Eli Lilly presentó la solicitud de patente 2.041.113, que protegía a olanzapina como una selección del genus de la patente 1.075.687. La patente señala que el compuesto sería utilizado como un antipsicótico

1. Sentencia del caso Eli Lilly y Compañía con Gobierno de Canadá, caso CIADI UNCT/14/2, 16 de marzo de 2017, páginas 94-95, párrafos 309-310. Disponible en http://bit.ly/2tEoyzM. 
para el tratamiento de condiciones mentales tales como la esquizofrenia. Finalmente, el 14 de julio de 1998, la patente de Zyprexa fue concedida.

El 2007, el laboratorio Novopharm obtuvo autorización comercial del Ministerio de Salud de Canadá para comercializar un medicamento genérico de Zyprexa. Ante esto, Eli Lilly presentó una demanda de infracción de su patente en contra de Novopharm. Posteriormente, el 10 de noviembre de 2011, la Corte Federal, junto con rechazar la demanda de infracción, resolvió que la patente era nula por no cumplir con el requisito de utilidad, pues Novopharm demostró que no se cumplió la promesa de utilidad de la patente y que no podía haber sido aplicada la alternativa, es decir, demostrar la utilidad mediante la predicción racional de la utilidad de acuerdo a la evidencia disponible en 1991 (fecha de su solicitud). Esta decisión fue apelada por Eli Lilly, pero el 10 de septiembre de 2012 la Corte Federal de Apelaciones rechazó dicho recurso. Luego, el 16 de mayo de 2013, la Corte Suprema decidió no revisar tal decisión.

La segunda patente de Eli Lilly, respecto de la cual recayó este proceso, fue la que protegía el medicamento Strattera, cuyo principio activo es atomoxetina, que ya formaba parte de un compuesto protegido por una patente en Canadá. El 4 de enero de 1996, Eli Lilly presentó una nueva solicitud de patente para un nuevo uso de la atomoxetina, la cual fue concedida el 1 de octubre de 2002 (patente 2.209.735). Esta patente es para el uso de la atomoxetina en un método de tratamiento del desorden psicológico de hiperactividad.

El laboratorio Novapharm presentó un recurso de nulidad en contra de la patente de Strattera. El 14 de septiembre de 2010, la Corte Federal resolvió que ésta era nula debido a que no cumplía el requisito de utilidad, pues si bien la utilidad estaba sustentada en la predicción racional, no cumplió con los requisitos de sustento y divulgación en la solicitud misma de la patente. Eli Lilly presentó un recurso de apelación, el cual fue rechazado por la Corte Federal de Apelaciones el 5 de julio de 2011. Finalmente, el 8 de diciembre de 2011, la Corte Suprema decidió no revisar el caso, por lo que la nulidad quedó firme y ejecutoriada.

\section{Inicio del arbitraje}

Producto de estas nulidades, Eli Lilly inició este arbitraje y realizó las siguientes peticiones: i) Canadá debía indemnizarlos por todos los daños emergentes y lucro cesante sufrido por el incumplimiento de sus obligaciones, de acuerdo al capítulo $17 \mathrm{del}$ TLCAN, lo cual estimó en una cifra no menor de 500 millones de dólares canadienses más otros montos adicionales; ii) costas judiciales; iii) intereses completos; iv) impuestos que se deben pagar respecto del monto de la indemnización, yv) cualquier otra indemnización que el Tribunal considerase justa y apropiada.

En base a lo propuesto por las partes, el Tribunal determinó que existían cinco te- 
mas que debían ser analizados: i) ¿cuál es la jurisdicción del Tribunal?; ii) ¿es la negación de justicia el único fundamento para sustentar la responsabilidad de un Estado por medidas judiciales de acuerdo al capítulo 11 del TLCAN?; iii) ¿existió un cambio drástico en la aplicación del requisito de la utilidad en la ley canadiense?; iv) ¿es el requisito de utilidad, en la forma en que fue aplicado a las patentes de Strattera y Zyprexa, arbitrario o discriminatorio?, y v) en caso de contestar en forma afirmativa los puntos iii) o iv), ¿fue la nulidad de las patentes de Zyprexa y de Strattera una violación de las obligaciones de Canadá de acuerdo a los artículos 1.105 y/o 1.110 del TLCAN?

A continuación, nos abocaremos a comentar los temas relacionados a la propiedad industrial y la utilidad que fueron analizados y resueltos por el Tribunal.

\section{El requisito de utilidad en Canadá y el supuesto cambio drástico en la ley canadiense}

El requisito de utilidad es esencialmente equivalente (aunque no idéntico) al requisito de aplicación industrial, el cual en Chile se encuentra definido en el artículo 36 de la Ley de Propiedad Industrial, que señala: «Se considera que una invención es susceptible de aplicación industrial cuando su objeto pueda, en principio, ser producido o utilizado en cualquier tipo de industria». La definición en la ley chilena sigue muy de cerca la de la mayoría de los países europeos, en los cuales «bastará con que la invención dé lugar a un objeto producible o que pueda ser utilizado en la producción de bienes o servicios para que sea patentable» (Cabanellas, 2004: 777).

El artículo 2 de la Patent Act (Ley de Patentes) de Canadá define a las invenciones como «cualquier arte, proceso, máquina, fabricación o composición de materia que sea nuevo y útil, o cualquier mejora nueva y útil». A su vez, el artículo 27 numeral 3 señala que «la memoria descriptiva de un invento debe describir en forma correcta y completa el invento y su operatividad o uso, tal como ha sido contemplado por su inventor». Sin embargo, la ley canadiense no define el requisito de utilidad.

Se ha entendido que el requisito de utilidad en Canadá se cumple si es que el invento demuestra un mero ápice de utilidad, lo que significa que es «susceptible de cumplir con su propósito", ${ }^{2}$ a menos que el solicitante realice una promesa de utilidad. En caso de que el solicitante realice una promesa, ésta debe ser demostrada expresamente o sustentada mediante una predicción racional de utilidad. La prueba y evidencia para demostrar la promesa o la predicción racional debe constar en la solicitud misma. En caso de que exista una promesa y no se realice, el invento no cumplirá con el requisito de utilidad, aunque lo haga con el estándar de un mero ápice de utilidad.

2. Wandscheer y otros con Sicard Ltd., Corte Suprema de Canadá, 22 de diciembre de 1947, foja 4. Disponible en http://bit.ly/2szYKZS. 
La doctrina legal canadiense ha definido la promesa como «una representación contenida en la memoria descriptiva de la patente, en forma tácita o expresa, de que el invento patentado logrará uno o más resultados deseables o evitará uno o más resultados no deseados» (Gold y Shortt, 2014: 38). Al respecto, la jurisprudencia ha señalado: «La promesa debe ser inferida por el juez de acuerdo a la patente íntegra, aplicando el criterio de una persona normalmente versada en el estado de la técnica al momento de la solicitud». ${ }^{3}$

En cuanto a la finalidad del requisito de utilidad en Canadá, autores como Kilic y Brooks han señalado que tiene dos funciones, a saber: i) determina la patentabilidad general del invento y ii) demuestra que un invento está concluido y que, por ende, puede entregar beneficios inmediatos al público, para lo cual una divulgación suficiente (es decir, cumplir la promesa) es necesaria. ${ }^{4}$

Habiendo revisado someramente el requisito de utilidad en Canadá, revisaremos a continuación la discusión y sentencia del Tribunal.

\section{El estándar de la promesa de utilidad}

Eli Lilly sostuvo que la doctrina de la promesa de utilidad es un cambio drástico respecto del estándar tradicional de utilidad en Canadá, al igual que respecto de los estándares de este requisito usados en Estados Unidos y México. Según el demandante, Canadá empleó durante décadas el estándar de requerir sólo un mero ápice de utilidad. Usando dicho estándar, las patentes de productos farmacéuticos nunca tuvieron problemas respecto del requisito de utilidad. ${ }^{5}$

Eli Lilly destacó que, en la década de los noventa, en Canadá se aplicaba un bajo estándar o umbral para determinar la utilidad. En cuanto a los inicios de la denominada doctrina de la promesa de la utilidad, Eli Lilly sostuvo que nació a mediados de la década del 200o, lo que produjo un cambio drástico al requisito de utilidad. Dicha doctrina tendría tres elementos, a saber: i) las autoridades deben identificar una «promesa» en la patente, que será el parámetro para medir la utilidad; ii) la prueba para demostrar la promesa no puede ser generada en forma posterior a la solicitud de la patente, y iii) las pruebas para sostener la utilidad sustentada en la predicción racional no son admisibles salvo que se incluyan en la misma solicitud de patente. ${ }^{6}$

3. Eli Lilly Canadá con Novopharm Limitada, Corte Federal de Apelaciones, 21 de julio de 2010, párrafo 93. Disponible en http://bit.ly/2tDJtG1.

4. Amicus curiae presentado por Burcu Kilic y Brook Baker del caso Eli Lilly y Compañía con Gobierno de Canadá, 23 de febrero de 2016, foja 1. Disponible en http://bit.ly/2rLLHSy.

5. Memoria de Eli Lilly del caso Eli Lilly y Compañía con Gobierno de Canadá, 29 de septiembre de 2014, foja 17, párrafo 36. Disponible en http://bit.ly/2qEuYUt.

6. Memoria de Eli Lilly del caso Eli Lilly y Compañía con Gobierno de Canadá, foja 27, párrafo 57, y foja 33, párrafo 70 . 
Al respecto, Canadá afirmó que este requisito no es nuevo y que tiene su origen en el caso de Consolboard de 1981, en la cual se señaló: «Si el invento no funciona, sea en el sentido de que simplemente no funciona para nada o, en un concepto más amplio, que no hará lo que la memoria descriptiva promete que hará».7

Además, Canadá sostuvo que el concepto de utilidad no está definido en la Patent Act y que corresponde a una evolución de la jurisprudencia. ${ }^{8}$ En particular, señaló que no existe una doctrina de la promesa de la utilidad, sino que son tres reglas separadas, una de las cuales es la promesa de utilidad. Canadá también destacó que existieron otras sentencias entre Consolboard y el año 2005 que aplicaron la regla de la promesa de utilidad (por ejemplo, Wellcome Foundation Ltd. con Apotex). ${ }^{9}$

El Tribunal resolvió que es difícil para ellos concluir que existió un cambio drástico de la ley respecto de este requisito, cuando las decisiones relevantes se extendieron por un período de años. El Tribunal también destacó que en los sistemas judiciales del Common Law es natural que la ley vaya evolucionando a través de las sentencias y que no se puede pretender que la ley sea absolutamente estática.

El Tribunal luego analizó la sentencia de Consolboard y resolvió que no acepta el argumento de Eli Lilly de que Consolboard nunca había sido citada por los tribunales en forma previa al año 2005 respecto de la regla de la promesa de utilidad. ${ }^{10} \mathrm{El}$ Tribunal también destacó la sentencia Wellcome de 1995 y el hecho de que existen sentencias posteriores al año 2005 en las cuales se citó Consolboard, específicamente respecto de la doctrina de la promesa de utilidad. ${ }^{11}$

El Tribunal concluyó que Consolboard es la decisión que establece el precedente judicial vinculante respecto de la existencia de la regla de la promesa de la utilidad (aunque no haya sido usado en forma frecuente por las cortes en Canadá). ${ }^{12}$ Por último, el Tribunal resolvió que no tiene razones para cuestionar las interpretaciones del Poder Judicial de Canadá respecto de sus propias decisiones. ${ }^{13}$

La doctrina canadiense parece estar de acuerdo con lo señalado por Canadá, en el sentido de que la denominada promesa de utilidad no sería una doctrina en sí, sino un conjunto de tres reglas que Eli Lilly decidió denominar como una doctrina. En relación a la promesa de utilidad, es claro que ella existía con anterioridad al año 2005, tal como sostuvo Canadá en su defensa y como fue confirmado por el Tribunal. La

7. Consolboard Inc. con MacMillan Bloedel (Sask.) Ltd., Corte Suprema de Canadá, 19 de marzo de 1981, foja 525. Disponible en http://bit.ly/2t4ti7m.

8. Sentencia del caso Eli Lilly y Compañía con Gobierno de Canadá, foja 81, párrafo 270.

9. Contramemoria del Gobierno de Canadá del caso Eli Lilly y Compañía con Gobierno de Canadá, 27 de enero de 2015, fojas 39-48, párrafos 88-107. Disponible en http://bit.ly/2rnGMaI.

10. Sentencia del caso Eli Lilly y Compañía con Gobierno de Canadá, foja 97, párrafo 318.

11. Sentencia del caso Eli Lilly y Compañía con Gobierno de Canadá, foja 97, párrafo 318.

12. Sentencia del caso Eli Lilly y Compañía con Gobierno de Canadá, foja 99, párrafo 324.

13. Sentencia del caso Eli Lilly y Compañía con Gobierno de Canadá, foja 98, párrafo 321. 
regla existe por lo menos desde la sentencia de Consolboard en 1981. Esto es sustentado por autores como Gold y Shortt, quienes señalaron al respecto que «la promesa de la utilidad es un concepto legal que tiene profundas raíces históricas y de alcance global» (Gold y Shortt, 2014: 37). Estos autores incluso señalan que Consolboard no fue el primer caso en el que se empleó el requisito de la promesa de la utilidad, sino que mencionan una sentencia previa, New Process Screw Corp. con PL Robertson Manufacturing Co. de 1961 (Gold y Shortt, 2014: 56). A su vez, la Canadian Generic Pharmaceutical Association, en su Amicus curiae de este caso, también identificó la sentencia del caso New Process Screw Corp. como la primera vez que la jurisprudencia se refirió a la regla de la promesa. ${ }^{14}$

\section{Prueba generada en forma posterior a la solicitud}

En relación a este tema, Eli Lilly sostuvo que dicho cambio drástico ocurrió en el año 2002 mediante la sentencia de la Corte Suprema en el caso Apotex Inc. con Wellcome Foundation Ltd., ${ }^{15}$ en la cual la Corte resolvió que la prueba generada en forma posterior a la fecha de presentación de la solicitud no es admisible para demostrar la utilidad del invento. ${ }^{16}$

Al respecto, Canadá señaló que Eli Lilly se equivocó al afirmar que esta regla cambió en virtud de la sentencia de Apotex, y sustentó su posición en los siguientes argumentos: i) el principio de que la utilidad debe ser establecida antes de la solicitud está consagrado en fallos previos, tales como Monsanto en 1979, que adoptó la doctrina de la predicción racional. Esta doctrina sería innecesaria si es que una patente pudiese ser concedida en base a puras especulaciones respecto de su utilidad, la que sólo podría ser confirmada mediante pruebas posteriores; ii) en Apotex nada sugiere que hubiere un cambio o elaboración de ley: la Corte Suprema en Apotex simplemente analizó la Patent Act y sentencias previas, en las cuales la Corte había requerido conocer la utilidad del invento al momento de presentar la solicitud, y iii) la comunidad jurídica no consideró que el caso Apotex fue un cambio drástico de la ley (por ejemplo, varios boletines de estudios jurídicos destacaron que la sentencia confirmó una regla existente y de antigua aplicación). ${ }^{17}$

Al respecto, el Tribunal resolvió que la sentencia de Apotex se fundó en la Patent Act, jurisprudencia previa y consideraciones de políticas públicas. En relación a la

14. Amicus curiae presentado por Jonathan Stainsby (Canadian Generic Pharmaceuticals Association) del caso Eli Lilly y Compañía con Gobierno de Canadá, 23 de febrero de 2016, foja 8. Disponible en http://bit.ly/2s5tMFP.

15. Apotex Inc. con Wellcome Foundation Ltd., Corte Suprema de Canadá, 5 de febrero de 2002, foja 153. Disponible en http://bit.ly/2sLLm3y.

16. Memoria de Eli Lilly del caso Eli Lilly y Compañía con Gobierno de Canadá, foja 32, párrafo 66.

17. Sentencia del caso Eli Lilly y Compañía con Gobierno de Canadá, fojas 84-86, párrafos 280-284. 
prueba presentada por Eli Lilly de otras decisiones previas que supuestamente habían permitido la presentación de pruebas y evidencias generadas con posterioridad a la fecha de la solicitud de la patente, resolvió que no eran concluyentes. ${ }^{18}$

En cuanto a las políticas públicas, este autor está de acuerdo con la lógica que sustenta este requisito en Canadá, pues tal como señaló la Corte Suprema de Estados Unidos en la sentencia de Brunner con Manson, «las patentes no pueden ser simples licencias de caza ni premios por la búsqueda de algo, sino que deben serlo por su exitosa conclusión». ${ }^{19}$

Otro tema interesante en este acápite es que, al revisar la prueba aportada por las partes y revisada por el Tribunal, resalta el hecho de que la comunidad jurídica (abogados y jueces) no interpretaron la sentencia de Apotex de manera uniforme, a tal nivel que varios boletines de estudios de propiedad industrial informaron a sus clientes que la sentencia confirmó la ley existente respecto de la fecha de la prueba de utilidad. Este autor considera interesante que el Tribunal haya analizado las opiniones específicas de los estudios jurídicos, por lo que creemos que sería una buena práctica que tanto el Instituto Nacional de Propiedad Industrial como el Ministerio de Relaciones Exteriores mantenga una bitácora de dicha información en caso de que en un futuro Chile sea parte en un juicio de estas características.

\section{La prueba de la predicción racional}

En relación a la prueba de la predicción racional de la utilidad, Eli Lilly sostuvo que la sentencia del caso Raloxifene en el 2008 implicó un cambio drástico a la ley, al establecer que los fundamentos de la predicción racional tenían que ser divulgados en la patente al momento de su solicitud. ${ }^{20}$

$\mathrm{Al}$ respecto, Canadá señaló que este requerimiento no es nuevo, y fundamentó su posición en las siguientes razones: i) la existencia de la divulgación de la predicción racional existe desde la sentencia de Monsanto de $1979 ;{ }^{21}$ ii) la sentencia Apotex confirmó la decisión de Monsanto en el sentido de tener que divulgar el fundamento de la predicción racional al momento de la solicitud, ${ }^{22}$ y iii) no existe precedente alguno en la ley que habría sustentado encontrar bases de una predic-

18. Sentencia del caso Eli Lilly y Compañía con Gobierno de Canadá, foja 102, párrafos 331 y 333.

19. Brenner con Manson, Corte Suprema de Estados Unidos, 21 de marzo de 1966. Disponible en http://bit.ly/2sLvoIb.

20. Memoria posterior a la audiencia de Eli Lilly del caso El Lilly y Compañía con Gobierno de Canadá, 25 de julio de 2016, foja 50, párrafo 113. Disponible en http://bit.ly/2rLTXll.

21. Memoria posterior a la audiencia del Gobierno de Canadá del caso Eli Lilly y Compañía con Gobierno de Canadá, foja 95, párrafo 153.

22. Memoria posterior a la audiencia del Gobierno de Canadá del caso Eli Lilly y Compañía con Gobierno de Canadá, 25 de julio de 2016, fojas 98-101, párrafos 155-158. Disponible en http://bit.ly/2tYnhpq. 
ción racional en relación a la patente de Strattera, que no divulgó información que pudiere evidenciarla. ${ }^{23}$

En relación a este tema, el Tribunal recordó que cuando la Corte Federal rechazó la apelación de Eli Lilly en el caso Raloxifene, dicha Corte fundó su decisión en el precedente de la decisión de Apotex, en la cual se había resuelto que para poder argumentar la predicción racional se tenían que cumplir varios requisitos, entre ellos, que debían existir fundamentos de hechos para poder emplear la predicción racional, y que el inventor tenía que incluir en la solicitud misma un razonamiento respecto de la predicción racional. ${ }^{24} \mathrm{El}$ Tribunal resolvió que el hecho de que la sentencia de $R a$ loxifene se fundó en un precedente judicial previo constituye una fuerte presunción en contra del demandante. ${ }^{25}$

Finalmente, el Tribunal resolvió que no puede aceptar la posición de Eli Lilly de que la sentencia de Raloxifene provocó un cambio drástico a la ley canadiense. El Tribunal estimó que existió un desarrollo progresivo durante décadas en relación a este requisito, que se inició con el caso Monsanto, fue mencionado en Apotex y finalmente aplicado directamente en el 2008 en la sentencia de Raloxifene. ${ }^{26}$

El primer comentario a realizar respecto de este tema es que existían precedentes judiciales en las sentencias de Monsanto y Apotex que mencionaron este requisito, por lo que difícilmente se puede considerar como nuevo. Además, en relación a la facultad de las cortes de ir precisando y realizando ciertos ajustes a las leyes, especialmente en países del Common Law, es necesario señalar que concordamos con la opinión del Tribunal de que esto ocurre con cierta frecuencia, tal como sucedió en las sentencias de la Corte Suprema de Estados Unidos en los casos de Myriad y de Alice.

\section{Comparación del requisito de utilidad con otras jurisdicciones}

Eli Lilly sostuvo que el cambio drástico respecto del requisito de utilidad también se puede observar al compararlo en Canadá con los otros Estados miembros del TLCAN, ${ }^{27}$ y destaca que en Estados Unidos y México existe un bajo estándar de utilidad. ${ }^{28}$ Eli Lilly fundó este argumento en el informe del profesor Robert Merges y en

23. Sentencia del caso Eli Lilly y Compañía con Gobierno de Canadá, foja 88, párrafo 290.

24. Sentencia del caso Eli Lilly y Compañía con Gobierno de Canadá, foja 105, párrafo 339.

25. Sentencia del caso Eli Lilly y Compañía con Gobierno de Canadá, foja 105, párrafo 340.

26. Sentencia del caso Eli Lilly y Compañía con Gobierno de Canadá, foja 108, párrafos 349-350.

27. Memoria posterior a la audiencia de Eli Lilly del caso Eli Lilly y Compañía con Gobierno de Canadá, foja 62, párrafo 136 .

28. Memoria posterior a la audiencia de Eli Lilly del caso Eli Lilly y Compañía con Gobierno de Canadá, foja 63, párrafo 139. Véase el informe de experto del Robert P. Merges del caso Eli Lilly y Compañía con Gobierno de Canadá, foja 4, párrafo 6. Disponible en http://bit.ly/2tDTwed. 
el informe especial 301 de la Oficina del Representante de Comercio de los Estados Unidos (USTR en inglés), que señaló que tenía importantes preocupaciones respecto del aumento en el estándar para demostrar la utilidad en Canadá. ${ }^{29}$

$\mathrm{Al}$ respecto, Canadá señaló que su requisito de utilidad no es tan diferente del que existe en Estados Unidos y México, especialmente considerando el requisito de reproducibilidad de Estados Unidos (enablement requirement). Asimismo, destacó que no existe un estándar propio en el derecho internacional respecto del requisito de utilidad.$^{30}$ En relación al informe especial 301 del USTR, Canadá señaló que no se funda en evidencia objetiva, sino en afirmaciones realizadas por distintas partes interesadas, incluyendo al mismo demandante, y que no ha sido objeto de ninguna otra crítica respecto de su requisito de utilidad por parte de otros países u organismos

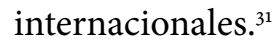

$\mathrm{Al}$ respecto, el Tribunal señaló que este argumento no parece ser relevante, ya que no entiende bien cómo una comparación con otras legislaciones es relevante para determinar si un país cambió drásticamente sus propias leyes..$^{32}$ En relación al informe especial 301 del USTR, el Tribunal señaló que es el único que se queja de la doctrina de la promesa de utilidad en Canadá y que el silencio de México y del resto de la comunidad internacional es más relevante que el contenido del informe. ${ }^{33}$ En definitiva, el Tribunal concluyó que el análisis comparativo con otras legislaciones no demuestra que existió un cambio drástico en las leyes de Canadá.

Respecto de este tema, Eli Lilly intentó convencer al Tribunal que al ser Canadá miembro del TLCAN, su ley tenía que tener el mismo estándar respecto de la utilidad que Estados Unidos y México (aplicación industrial en el caso de este último). Ahora, si se revisa el artículo 1.709 numeral 1 del TLCAN, es evidente que utiliza como base la misma redacción que el Acuerdo sobre los Aspectos de los Derechos de Propiedad Intelectual relacionados con el Comercio (ADPIC) en su artículo 27 numeral 1. Sin embargo, éste debe ser analizado en conjunto con el artículo 1 del mismo instrumento, que señala: «Los Miembros podrán establecer libremente el método adecuado para aplicar las disposiciones del presente Acuerdo en el marco de su propio sistema y práctica jurídica», por lo que, efectivamente, los países tienen ciertas flexibilidades en relación a la definición y determinación de dichos requisitos.

29. Memoria posterior a la audiencia de Eli Lilly del caso Eli Lilly y Compañía con Gobierno de Canadá, foja 75, párrafo 155 .

30. Contramemoria del Gobierno de Canadá del caso Eli Lilly y Compañía con Gobierno de Canadá, fojas 78-85, párrafos 181-199.

31. Memoria posterior a la audiencia del Gobierno de Canadá del caso Eli Lilly y Compañía con Gobierno de Canadá, foja 111, párrafo 174.

32. Sentencia del caso Eli Lilly y Compañía con Gobierno de Canadá, foja 117, párrafo 377.

33. Sentencia del caso Eli Lilly y Compañía con Gobierno de Canadá, foja 117, párrafo 378. 
Esta posición también ha sido sostenida por juristas como Jerome Reichmann, quien ha escrito respecto del ADPIC que

estableció un set de estándares internacionales mínimos de protección para los 150 países que participaron. Sin embargo, el Acuerdo, que no intentó crear un régimen de patentes global uniforme o profundamente armonizado, dejó amplias posibilidades para las variaciones y aplicaciones nacionales (Reichmann y Dreyfuss, 2007: 89).

A su vez, Billingsley destaca que la Conferencia de las Naciones Unidas respecto de Comercio y Desarrollo señaló respecto del artículo 27 numeral 1 del ADPIC que «establece criterios de patentabilidad; sin embargo, no armoniza la forma en la cual ellos deben ser aplicados. Por ende, los Miembros tienen considerable libertad de acción en la aplicación de dichos tres requisitos (novedad, altura inventiva y aplicación industrial)» (Billingsley, 2015: 32).

Asimismo, resulta bastante clara la opinión de los profesores Kilic y Baker, quienes en su Amicus curiae señalaron:

De acuerdo al TLCAN y otros acuerdos análogos, incluyendo el ADPIC, los países mantienen considerables flexibilidades para establecer sus propios estándares de patentabilidad siempre que se cumplan con los mínimos básicos acordados [...] el TLCAN no especifica cómo estos criterios deben ser definidos ni aplicados. ${ }^{34}$

Esta posición ha sido compartida por otros reconocidos profesores, tales como Valentina Vadi (2016: 120) y Grosse, Liddell y Waibel. ${ }^{35}$

Incluso, la jurisprudencia en el Common Law ha resuelto que los requisitos de patentabilidad, tales como la utilidad, no se encuentran armonizados. En este sentido, debemos destacar la sentencia de la Corte Suprema del Reino Unido en el caso Human Genome Sciences Inc. con Eli Lilly y Compañia, en la cual resolvió:

Particularmente, cuando se trata de una cuestión interesante como la delimitación precisa de las fronteras entre la patentabilidad y la falta de patentabilidad por motivos de aplicación industrial, no sería sorprendente que la Ley no fuera idéntica en las dos jurisdicciones. ${ }^{36}$

Asimismo, esta conclusión también ha sido adoptada por la misma Organización Mundial de la Salud, la Organización Mundial de Comercio y la Organización

34. Amicus curiae presentado por Burcu Kilic y Brook Baker del caso Eli Lilly y Compañía con Gobierno de Canadá, foja 1.

35. Amicus curiae presentado por Henning Grosse Ruse-Khan, Kathleen Liddell y Michael Waibel del caso Eli Lilly y Compañía con Gobierno de Canadá, 12 de febrero 2016, foja 9. Disponible en http:// bit.ly/2sLRmcA.

36. Human Genome Sciences Inc. con Eli Lilly y Compañía, Corte Suprema del Reino Unido, 2 de noviembre de 2011, párrafo 41. Disponible en http://bit.ly/2rMb2vw. 
Mundial de la Propiedad Industrial, las que en su informe conjunto del año 2012 señalaron:

Aunque en la gran mayoría de los países se encuentran los mismos criterios esenciales de patentabilidad, no existe acuerdo internacional sobre la definición e interpretación de estos criterios. Esto otorga un margen de política con respecto a su establecimiento de acuerdo a la ley nacional aplicable. En consecuencia, las oficinas de patentes y los tribunales interpretan y aplican los requisitos nacionales de patentabilidad caso por caso dentro del marco jurídico aplicable (OMC, OMPI y OMS, 2012: 57).

Por estas razones, nos parece correcto que el Tribunal no aceptara el argumento de Eli Lilly de que los requisitos de patentabilidad se encuentran armonizados a nivel internacional.

\section{Expectativas legítimas de Eli Lilly}

Eli Lilly afirmó que la compañía tenía una expectativa legítima de que las patentes de Strattera y Zyprexa no serían invalidadas en virtud del requisito de utilidad. ${ }^{37}$ Esto se fundaba en la Patent Act al momento de la solicitud, el hecho de que las patentes de Strattera y Zyprexa fueron concedidas y las obligaciones de Canadá de acuerdo al TLCAN. ${ }^{8}$ Asimismo, Eli Lilly sostuvo que Canadá, al conceder las patentes, realizó un compromiso con ellos que les aseguraba que gozarían del derecho excluyente para fabricar, usar y comercializar su invento..$^{39}$ Finalmente, Eli Lilly señaló que ellos también confiaron en el cumplimiento de parte de Canadá de sus obligaciones internacionales, incluyendo el capítulo 17 del TLCAN, el que establecía que Canadá tenía la obligación de no desarrollar y aplicar en forma retroactiva una doctrina como la promesa de utilidad. ${ }^{40}$ Así, el cambio drástico del requisito de utilidad se encontraría fuera del «margen de cambio aceptable» que los inversionistas deben anticipar.

En relación a este punto, Canadá destacó que el sólo hecho de no cumplir con las expectativas del demandante no implica una infracción al estándar mínimo de tratamiento establecido en el artículo 1.105 numeral 1 del TLCAN. ${ }^{41}$ Asimismo, Canadá destacó que para que exista esta expectativa legitima, ésta debe fundarse en hechos

37. Memoria de respuesta de Eli Lilly del caso Eli Lilly y Compañía con Gobierno de Canadá, 11 de septiembre de 2015, foja 174, párrafo 349. Disponible en http://bit.ly/2rXf6w6.

38. Memoria de respuesta de Eli Lilly del caso Eli Lilly y Compañía con Gobierno de Canadá, sección V letra b) número 2, foja 175, párrafos 349 y siguientes.

39. Memoria de Eli Lilly del caso Eli Lilly y Compañía con Gobierno de Canadá, foja 137, párrafo 285.

40. Memoria de Eli Lilly del caso Eli Lilly y Compañía con Gobierno de Canadá, foja 134, párrafo 279.

41. Contramemoria del Gobierno de Canadá del caso Eli Lilly y Compañía con Gobierno de Canadá, foja 121, párrafo 276 , foja 125, párrafo 283 . 
objetivos e incluir una promesa específica realizada por el Estado. Canadá señaló que nunca realizó ninguna clase de promesa al demandante en relación a sus patentes para Strattera y Zyprexa, y que el demandante sabía que sus patentes podían ser objeto de procesos de nulidad. ${ }^{42}$

Al respecto, el Tribunal señaló que este argumento del demandante se sustenta en el artículo 1.105 del TLCAN y que, por ende, depende de que el demandante demuestre que existió un cambio drástico en la ley canadiense que estaba fuera del «margen de cambio aceptable» respecto del requisito de utilidad. Siendo que no lo demostró, entonces simplemente no puede haber afectado sus expectativas legítimas. ${ }^{43}$

El Tribunal también destacó que el demandante sabía que los inventos respecto de los cuales se solicita una patente deben cumplir con ciertos requisitos, tales como la utilidad; también tenía conocimiento de que las patentes pueden ser anuladas en Canadá por no cumplir con alguno de dichos requisitos. A mayor abundamiento, el Tribunal, tal como hemos mencionado anteriormente, consideró que las tres reglas de la doctrina de la promesa de la utilidad tenían sustento en la ley canadiense al momento de las solicitudes de las patentes para Strattera y Zyprexa, ${ }^{44}$ debido a que los procesos de sus nulidades se sustentaron en la ley vigente y no afectaron las expectativas legitimas del demandante.

En relación a las expectativas legítimas que un titular de una patente puede tener, nos parece evidente que los titulares de patentes de invención saben que éstas pueden ser objeto de demandas de nulidad. Eli Lilly debía tomar en consideración el artículo 1.709 numeral 8 del TLCAN, el cual establece que pueden existir procedimientos de nulidad de patentes de invención, al igual que el artículo 60 numeral 1 de la Patent Act de Canadá, que establece la existencia del procedimiento de nulidad. Por ende, es difícil aceptar una posición que afirma que se afectaron sus expectativas legítimas si se empleó un procedimiento claro fundado en reglas existentes al momento de las solicitudes.

A mayor abundamiento, las patentes de invención no otorgan derechos positivos sino negativos, es decir, no otorgan el derecho a usar, comercializar, importar o exportar un producto, sino sólo a evitar que terceros lo hagan. Tal como señala Vadi, las patentes «no confieren derechos absolutos ni crean ninguna expectativa legítima de que la exclusividad que confieren es absoluta y seguirá sin interferencia de los controles y balances aceptados e inherentes al sistema de propiedad intelectual» (Vadi, 2016: 171).

Nos parece correcta la decisión del Tribunal al indicar que, si una patente es anu-

42. Contramemoria del Gobierno de Canadá del caso Eli Lilly y Compañía con Gobierno de Canadá, fojas 129, párrafo 294.

43. Sentencia del caso Eli Lilly y Compañía con Gobierno de Canadá, foja 118, párrafo 380.

44. Sentencia del caso Eli Lilly y Compañía con Gobierno de Canadá, foja 118, párrafo 384. 
lada, no se afectan las expectativas legítimas de su titular en la medida que el proceso de nulidad haya cumplido con los requisitos legales correspondientes y que haya existido un debido proceso.

\section{Conclusiones generales del Tribunal}

El Tribunal resolvió que el requisito de utilidad de la Ley canadiense sufrió una evolución incremental y gradual entre el momento en que las patentes para Zyprexa y Strattera fueron concedidas y cuando fueron anuladas, especialmente durante el período de 2002 a 2008. En dicho período hubo un aumento en el número de casos en que la utilidad fue el fundamento para cuestionar la validez de patentes farmacéuticas, lo que al parecer generó un incremento en el ritmo de evolución de la ley aplicable a ese sector. Además, el Tribunal consideró que se había demostrado que los tres requisitos que el demandante sostiene que forman parte de la doctrina de la promesa de utilidad tienen un fundamento razonablemente sólido en sentencias y leyes previas, independiente de si ellas fueron aplicadas o no en forma constante con anterioridad. Por lo tanto, el Tribunal concluyó que no existió un cambio drástico en el requisito de utilidad de la ley canadiense.

A pesar de que el Tribunal resolvió que esto último es suficiente para desestimar la demanda, consideró ${ }^{45}$ que también debe analizar la supuesta naturaleza arbitraria y discriminatoria del requisito de utilidad en Canadá, con el objetivo de que el fallo sea absolutamente completo y evitar cualquier posibilidad de que siquiera exista un atisbo de duda respecto de sus conclusiones.

\section{La supuesta naturaleza arbitraria y discriminatoria del requisito de utilidad en Canadá}

\section{Arbitrariedad}

Eli Lilly sostuvo que la doctrina de la promesa de la utilidad es arbitraria por las siguientes razones: i) es impredecible e incoherente y ii) no cumple ninguna política pública legítima. ${ }^{46}$

Eli Lilly afirmó que la doctrina es impredecible e incoherente, pues el proceso varía de caso en caso y de corte en corte, por lo que no existe un criterio uniforme. ${ }^{47}$ En relación al aumento de la carga probatoria, el demandante sostuvo que ésta fue arbitraria, pues no permite la presentación de pruebas posteriores a la fecha de la solicitud de la patente que demuestren la validez de evidencias anteriores a dicha fecha

45. Sentencia del caso Eli Lilly y Compañía con Gobierno de Canadá, foja 120, párrafo 389.

46. Sentencia del caso Eli Lilly y Compañía con Gobierno de Canadá, foja 120, párrafo 390.

47. Memoria de Eli Lilly del caso Eli Lilly y Compañía con Gobierno de Canadá, foja 125, párrafo 263. 
que verificasen la probable efectividad de una droga.$^{4} \mathrm{El}$ demandante sostiene que la regla pone a las empresas farmacéuticas en un dilema, pues si invierten el tiempo en completar los ensayos clínicos correspondientes (fases preclínicas y clínicas), pueden ya no cumplir con los requisitos de novedad y de altura inventiva de la patente..$^{49}$

Finalmente, Eli Lilly sostuvo que la doctrina de la promesa de utilidad no cumple con ninguna finalidad legítima de políticas públicas. ${ }^{50}$

$\mathrm{Al}$ respecto, junto con reiterar que no existe tal doctrina, sino varias reglas de la Ley de Patentes, Canadá afirmó que es falso que el proceso de construcción e interpretación de la promesa de utilidad sea inherentemente impredecible..$^{51}$ En relación a la afirmación del demandante de que los jueces deben «explorar» las patentes para encontrar promesas, señaló que, de hecho, los jueces deben decidir en base a la evidencia presentada por las partes. En ese sentido, los distintos resultados se deben a la alta dependencia en hechos de los juicios, que difieren de caso a caso. ${ }^{52}$ En cuanto a las políticas públicas, Canadá argumentó que no existe nada arbitrario en exigir que las patentes demuestren sus promesas de utilidad ${ }^{53}$ ni que divulguen el sustento de su predicción racional de utilidad en la solicitud de patente, sino que ello es una parte esencial del acuerdo entre el titular de la patente y la sociedad. ${ }^{54}$

En relación a la prohibición de presentación de pruebas posteriores a la fecha de la solicitud, Canadá sostuvo que no es arbitrario exigirles a los solicitantes que demuestren o empleen la predicción racional de la utilidad de la patente al momento de su presentación..$^{55}$ En cuanto a la afirmación del demandante de que este requisito es arbitrario contra las patentes farmacéuticas, Canadá señaló que no es requisito presentar ensayos clínicos y que es sabido que en forma constante se conceden patentes farmacéuticas sustentadas en estudios realizados sobre animales (fase preclínica)..$^{56}$

El Tribunal resolvió que Eli Lilly no demostró que la práctica de encontrar la promesa en las reivindicaciones y en la memoria descriptiva de la solicitud sea im-

48. Memoria posterior a la audiencia de Eli Lilly del caso Eli Lilly y Compañía con Gobierno de Canadá, foja 148, párrafo 301.

49. Memoria de Eli Lilly del caso Eli Lilly y Compañía con Gobierno de Canadá, foja 127, párrafo 266.

50. Sentencia del caso Eli Lilly y Compañía con Gobierno de Canadá, foja 122, párrafo 395.

51. Memoria posterior a la audiencia del Gobierno de Canadá del caso Eli Lilly y Compañía con Gobierno de Canadá, foja 16o, párrafo 244.

52. Sentencia del caso Eli Lilly y Compañía con Gobierno de Canadá, foja 125, párrafo 404.

53. Memoria posterior a la audiencia del Gobierno de Canadá del caso Eli Lilly y Compañía con Gobierno de Canadá, fojas 161-162, párrafo 246.

54. Memoria posterior a la audiencia del Gobierno de Canadá del caso Eli Lilly y Compañía con Gobierno de Canadá, foja 172, párrafo 263.

55. Memoria posterior a la audiencia del Gobierno de Canadá del caso Eli Lilly y Compañía con Gobierno de Canadá, foja 166, párrafo 252.

56. Memoria posterior a la audiencia del Gobierno de Canadá del caso Eli Lilly y Compañía con Gobierno de Canadá, foja 171, párrafo 260. 
predecible e incoherente; por el contrario, el proceso interpretativo realizado por las cortes canadienses está dentro del ámbito de sus facultades, por lo que algún nivel de imprevisibilidad es esperable en la aplicación de la ley. ${ }^{57}$

Además, resolvió que Canadá demostró que la aplicación de la doctrina de la promesa de la utilidad tiene una finalidad sustentada en políticas públicas legítimas. En específico, concuerda con la afirmación de Canadá de que la exigencia de la promesa en las reivindicaciones y la memoria descriptiva permite asegurar que el público obtiene su justa retribución del acuerdo de la patente y de que su contenido sea preciso..$^{58}$

En relación a la prohibición de presentación posterior de pruebas respecto de la utilidad, el Tribunal resolvió que el demandante no demostró que dicha regla fuese impredecible o incoherente, y que establece una clara fecha en la cual el solicitante debe demostrar la utilidad de la patente. ${ }^{59}$ Asimismo, el Tribunal aceptó el argumento de Canadá de que dicho plazo fue establecido al momento de su solicitud para evitar la concesión de patentes en base a pura especulación. ${ }^{60}$

En relación al argumento sobre las complejidades de demostrar el requisito de utilidad al momento de presentar la solicitud, el Tribunal no desconoció que puede ser complejo definir el cumplimiento de los requisitos de patentabilidad para presentar la solicitud de patente en el caso de muchos productos potenciales en el campo farmacéutico, pero considera que es una política pública racional y que aquello no es arbitrario. ${ }^{61}$

En relación al requisito de tener que incluir el sustento de la predicción racional de la utilidad al momento de la solicitud, el Tribunal resolvió que Canadá presentó argumentos para sustentar este requisito, siendo que la predicción racional permite que solicitantes puedan cumplir con el requisito aun antes de poder demostrar la utilidad del invento. ${ }^{62}$ En definitiva, el Tribunal resolvió que Canadá no incurrió en ningún acto arbitrario.

Estimamos que algunos de los argumentos de Eli Lilly en esta sección podrían tener cierto grado de sustento. Así, la eventual demora en la cual pueden incurrir los laboratorios farmacéuticos al presentar una solicitud, producto de la necesidad de recolectar la información necesaria para cumplir con la promesa de la utilidad, efectivamente puede afectar su capacidad para cumplir con los requisitos de novedad y altura inventiva. Si al solicitante se le piden los resultados de los ensayos preclínicos o clínicos para demostrar la eficacia y seguridad del producto farmacéutico (los

57. Sentencia del caso Eli Lilly y Compañía con Gobierno de Canadá, foja 131, párrafos 420-421.

58. Sentencia del caso Eli Lilly y Compañía con Gobierno de Canadá, fojas 131-132, párrafo 423.

59. Sentencia del caso Eli Lilly y Compañía con Gobierno de Canadá, foja 132, párrafo 424.

6o. Sentencia del caso Eli Lilly y Compañía con Gobierno de Canadá, foja 132, párrafo 425.

61. Sentencia del caso Eli Lilly y Compañía con Gobierno de Canadá, foja 132, párrafo 426.

62. Sentencia del caso Eli Lilly y Compañía con Gobierno de Canadá, foja 133, párrafo 428. 
primeros duran un par de años, los segundos entre cuatro y nueve años), es posible que el retraso en la presentación de la patente debido a que fue necesario esperar por dichos resultados afecte la posibilidad de que la solicitud de patente pueda cumplir con los requisitos de novedad y altura inventiva. En este mismo sentido se pronunciaron PhRMA, AMIIF y BIO en su Amicus curiae presentado en este caso, en el cual señalaron:

La presión [que la promesa de la utilidad] impone al dilatar la presentación de la patente (mientras se recolecta la información necesaria) aumenta en forma substancial el riesgo de que la innovación de la patente será negada en base a los requisitos de novedad o de ausencia de altura inventiva. ${ }^{63}$

Señalado lo anterior, estamos de acuerdo con la posición de Canadá, aceptada por el Tribunal, respecto de los fundamentos de políticas públicas sobre la regla de la promesa de utilidad. En este sentido, la doctrina canadiense ha señalado que ella cumple tres fines:

i) que el solicitante sea responsable por el beneficio público prometido en la patente a cambio del monopolio legal que obtendrá a través de la patente; ii) asegurarse que el solicitante haya realizado suficiente investigación y desarrollo para entender y comunicar cómo el invento funciona de acuerdo a sus reivindicaciones; iii) prevenir las dobles patentes, especialmente respecto de las patentes de selección (Gold y Shortt, 2014: 38).

En este sentido, siempre es bueno recordar, tal como señala la profesora Valentina Vadi, que «el sistema de patente se funda en el intercambio entre la promoción de la creación de conocimiento y la difusión del conocimiento» (Vadi, 2016: 120). Esta difusión sólo se puede realizar en forma íntegra si la patente incluye toda la información pertinente y correspondiente, pues es la única forma en que la sociedad tomará conocimiento cabal del invento.

\section{Discriminación}

Eli Lilly sostuvo que la doctrina de la promesa de utilidad discrimina contra las patentes farmacéuticas como un campo de la tecnología, y ello está expresamente prohibido en el artículo 1.709 numeral 7 del TLCAN. ${ }^{64}$

63. Amicus curiae presentado por Dianne Elderkin, Alan Yanovich, Matthew Scarola y Pratik Shah (Pharmaceutical Research and Manufacturers of America, PhRMA; Mexican Association of the Research based Pharmaceutical Industry, AMIF, y Biotechnology Innovation Organization, BIO) del caso Eli Lilly y Compañía con Gobierno de Canadá, 12 de febrero de 2016. Disponible en http://bit.ly/2tEioE5.

64. Memoria de Eli Lilly del caso Eli Lilly y Compañía con Gobierno de Canadá, fojas 101 y siguientes, párrafos 216-226. 
Eli Lilly sostuvo que, si bien a priori la doctrina de la promesa de la utilidad puede no parecer discriminatoria en contra de las patentes farmacéuticas, la evidencia empírica demuestra que sí discrimina contra este campo de la tecnología ${ }^{65}$ por las siguientes razones: i) antes del 2005, ninguna patente farmacéutica fue anulada por ausencia de utilidad; ${ }^{66}$ ii) desde el 2005, las cortes han resuelto que más de veintitrés patentes farmacéuticas no cumplen con el requisito de utilidad, pero no han resuelto lo mismo respecto de patentes de invención en otros campos de la tecnología, ${ }^{67}$ y iii) desde el 2005, el $41 \%$ de las decisiones respecto del requisito de utilidad han resuelto que una patente de invención del campo farmacéutico carece de dicho requisito, mientras que respecto de otros campos de la tecnología el porcentaje es o $\%{ }^{68}$

Eli Lilly también sostuvo que es posible concluir que el demandado ha tenido la intención expresa de discriminar en contra de las patentes farmacéuticas ${ }^{69} \mathrm{y}$ funda esta afirmación en los siguientes hechos: i) la doctrina de la promesa de utilidad fue adoptada por las cortes en litigios respecto de patentes farmacéuticas; ${ }^{70}$ ii) la Corte Federal ha resuelto en la parte dispositiva de su sentencia en Pfizer Canadá Inc. con Pharmascience Inc. que el fundamento para la predicción racional, al menos respecto de las patentes farmacéuticas, debe ser divulgado en la patente; ${ }^{71}$ iii) existe un estándar más alto para demostrar la utilidad en las patentes farmacéuticas que en otros campos tecnológicos como en la mecánica, en el que la utilidad puede demostrarse mediante un modelo, sin testeos, ${ }^{72} \mathrm{y}$ iv) el requisito de la doctrina entra en conflicto con la realidad del desarrollo de drogas innovadoras. ${ }^{73}$

Eli Lilly también afirmó que ha existido discriminación en relación a la nacionalidad de los solicitantes y titulares de las patentes rechazadas o anuladas por ausencia

65. Memoria posterior a la audiencia de Eli Lilly del caso Eli Lilly y Compañía con Gobierno de Canadá, foja 123, párrafo 257 .

66. Memoria de respuesta de Eli Lilly del caso Eli Lilly y Compañía con Gobierno de Canadá, foja 146, párrafo 294

67. Memoria de Eli Lilly del caso Eli Lilly y Compañía con Gobierno de Canadá, foja 103 y siguientes, párrafo 221.

68. Memoria de Eli Lilly del caso Eli Lilly y Compañía con Gobierno de Canadá, foja 104 y siguientes, párrafo 222.

69. Memoria de Eli Lilly del caso Eli Lilly y Compañía con Gobierno de Canadá, foja 104 y siguientes, párrafos 223-235.

70. Sentencia del caso Eli Lilly y Compañía con Gobierno de Canadá, foja 124, párrafo 400.

71. Memoria de Eli Lilly del caso Eli Lilly y Compañía con Gobierno de Canadá, foja 104 y siguientes, párrafo 224.

72. Memoria de Eli Lilly del caso Eli Lilly y Compañía con Gobierno de Canadá, foja 104 y siguientes, párrafo 224.

73. Memoria de Eli Lilly del caso Eli Lilly y Compañia con Gobierno de Canadá, foja 105 y siguientes, párrafo 225. 
de utilidad, pues en todos los casos han sido de titulares extranjeros, mientras que el gran beneficiario ha sido la industria farmacéutica genérica canadiense. ${ }^{74}$

Respecto de este tema, Canadá sostuvo que el mismo demandante ha reconocido que la doctrina de la promesa de utilidad no es discriminatoria a priori contra las patentes farmacéuticas. ${ }^{75}$ Además, destacó que, medidos por las ventas, la mitad de los dieciocho laboratorios genéricos más grandes en Canadá son extranjeros y que el requisito también se aplica a laboratorios innovadores canadienses. ${ }^{76}$

El Tribunal señaló que la doctrina de la promesa de la utilidad no produjo un efecto desventajoso en el campo farmacéutico en forma discriminatoria. ${ }^{77}$ Además, indicó que el demandante no pudo probar una relación de causalidad entre la doctrina de la promesa de la utilidad y la alta invalidación de patentes en el sector farmacéutico. El Tribunal señaló en relación a la sentencia del caso Pfizer Canadá Inc. que no puede resolver que dicha sentencia demuestre que existe discriminación respecto del ámbito farmacéutico, pues el caso se refería precisamente a una patente farmacéutica. ${ }^{7}$

Siendo que la denominada doctrina de la promesa de la utilidad se aplica en Canadá a todas las patentes, independiente de su campo tecnológico, parece difícil sustentar que dicha doctrina discrimina exclusivamente en contra del campo farmacéutico (más allá de que el requisito es complejo para este campo). En relación a la discriminación por nacionalidad, el hecho de que los laboratorios genéricos más grandes en Canadá sean extranjeros también parece desvirtuar esta línea de argumentos del demandante.

\section{Conclusiones generales finales del Tribunal}

El Tribunal resolvió que Eli Lilly no demostró las premisas de sus peticiones y argumentos (no existió un cambio drástico en la ley) y consideró que las nulidades de las patentes de Zyprexa y Strattera, a través de reglas que el demandante ha denominado como la doctrina de la promesa de la utilidad, no constituyen una expropiación en base al artículo 1.11o del TLCAN o una violación de los estándares mínimos de tratamiento del artículo 1.105 del tratado. El Tribunal también concluyó que no existió un acto arbitrario o discriminatorio de parte de Canadá en contra del demandante. Por estas razones, el Tribunal rechazó la demanda de Eli Lilly. ${ }^{79}$

74. Memoria de Eli Lilly del caso Eli Lilly y Compañía con Gobierno de Canadá, foja 105, párrafo 226.

75. Sentencia del caso Eli Lilly y Compañía con Gobierno de Canadá, foja 127, párrafo 409.

76. Sentencia del caso Eli Lilly y Compañía con Gobierno de Canadá, foja 129, párrafo 415.

77. Sentencia del caso Eli Lilly y Compañía con Gobierno de Canadá, foja 134, párrafos 432-433.

78. Sentencia del caso Eli Lilly y Compañía con Gobierno de Canadá, foja 136, párrafo 438.

79. Sentencia del caso Eli Lilly y Compañía con Gobierno de Canadá, foja 146, párrafo 469. 


\section{Comentarios finales}

A continuación, realizaré algunos breves comentarios finales relacionados tanto con el derecho de patentes como con otras áreas del derecho.

El primero es que si bien el Tribunal no se pronunció en forma expresa respecto del alcance del concepto de negación de justicia ${ }^{80}$ en el TLCAN, y si es que éste es la única forma en que un país será responsable internacionalmente por las decisiones de sus cortes (fue una defensa presentada por Canadá), el Tribunal sí mencionó que el Poder Judicial es un órgano del Estado y que, en principio, sus decisiones sí son atribuibles a dicho Estado. Por lo tanto, es posible contemplar circunstancias en las cuales una decisión judicial de una corte conlleva a la responsabilidad internacional de un Estado de acuerdo al TLCAN. Un ejemplo de esto serían las sentencias flagrantemente arbitrarias o discriminatorias que implicaran un cambio drástico en la ley.

Segundo, el Tribunal no analizó expresamente si el cambio en la ley canadiense constituía una expropiación regulatoria, sino que simplemente resolvió que Eli Lilly no logró demostrar que este cambio drástico ocurrió en los hechos, y que, por ende, no podía constituir una acción expropiatoria de acuerdo al artículo 1.110 del TLCAN. Por lo tanto, alguien podría sostener que, si hubiese existido un cambio drástico en la ley, ello podría constituir una expropiación regulatoria.

Tercero, y en relación al verdadero interés de Eli Lilly, si bien es indiscutible que las patentes sobre sus productos Strattera y Zyprexa son de un gran valor, pareciese ser que su verdadero interés iba más allá, es decir, forzar una modificación en la denominada doctrina de la promesa de utilidad, la cual se ha convertido en un requisito de cierta complejidad para cumplir en las patentes farmacéuticas de Canadá.

Cuarto, este autor estima que el hecho de que el Tribunal no le haya dado mayor preponderancia al informe especial 301 del USTR es una conclusión relevante para Chile, considerando que en el último tiempo dicho informe no ha calificado bien el cumplimiento del país en sus obligaciones relacionadas con la propiedad industrial.

Quinto, teniendo en cuenta que el derecho de propiedad industrial tiene elementos subjetivos por esencia, tales como la altura inventiva, este autor estima que es esencial destacar el hecho de que el Tribunal resolvió que el derecho puede ir evolucionado dentro de un margen aceptable (incluso si lo señala respecto de un sistema del Common Law). Esta rama del derecho está relacionada en forma estrecha con los avances tecnológicos, por lo que, en la medida en que la tecnología evolucione, es importante que los países tengan la posibilidad de ir realizando los ajustes necesarios y que éstos cumplan con las necesidades del país.

Finalmente, y considerando que en los últimos años la mayoría de los Tratados de Libre Comercio han elevado la protección de la propiedad industrial e intelectual al

8o. Sentencia del caso Eli Lilly y Compañía con Gobierno de Canadá, foja 64, párrafo 220. 
nivel de inversiones protegidas (aunque se discute si una mera solicitud de un derecho es suficiente para presentar una demanda ${ }^{81}$ y que ya se ha resuelto que la cancelación arbitraria de registros sanitarios son una expropiación indirecta, ${ }^{82}$ parece ser una conclusión racional que en los próximos años existirá un aumento de arbitrajes comerciales internacionales respecto de temas de propiedad industrial e intelectual. Habiendo señalado lo anterior, a la fecha no son pocos los casos en los cuales estos arbitrajes han sido resueltos a favor de las posiciones de los países.

\section{Referencias}

Billingsley, James (2015). «Eli Lilly and Company v The Government of Canada and the perils of investor-State arbitration». Journal University of Victoria, 20: 2741. Disponible en http://bit.ly/2sidofO.

Cabanellas, Guillermo (2004). Derecho de las patentes de invención. 2. a ed. Tomo I. Buenos Aires: Heliasta.

Gold, Richard y Michael SHORTT (2014). "The promise of the patent in Canada and around the world». Canadian Intellectual Property Review, 30 (1): 35-81. Disponible en http://ssrn.com/abstract=2361146.

OMC, Organización Mundial del Comercio, Organización Mundial de Propiedad Intelectual y Organización Mundial de la Salud (2012). Promoting access to medicine innovation and technology: intersections between health, intellectual property and trade. Disponible en http://bit.ly/2tYzo5R.

Reichman, Jerome y Rochelle Dreyfuss (2007). «Harmonization without consensus: critical reflections on drafting a substantive patent law treaty». Duke Law Journal, 57 (1): 85-130. Disponible en http://bit.ly/2rM8flZ.

VADI, Valentina (2016). «Towards a new dialectics: pharmaceutical patents, public health and foreign direct investments». Journal of Intellectual Property and Entertainment Law, 5 (1): 113-195. Disponible en http://bit.ly/2sLsRvP.

\section{Sobre el autor}

Cristián Barros Michell es abogado. Licenciado en Ciencias Jurídicas y Sociales por la Universidad Diego Portales. LL.M., Duke University, School of Law. Su correo electrónico es ckbarrosm@gmail.com.

81. Sentencia del caso Apotex Holdings Inc. con Estados Unidos, CIADI caso ARB(AF)/12/1, 25 de agosto de 2014, foja 174. Disponible en http://bit.ly/2rMnM56.

82. Sentencia del caso Les Laboratoires Servier, S.A.S. con República de Polonia, UNCITRAL, 14 de febrero de 2012. Disponible en http://bit.ly/2t4vsny. 